


\title{
Fichte o Deleuze, ¿quién es el dogmático?
}

\section{Fichte or Deleuze, who is the dogmatic?}

\author{
Julián Ferreyra \\ CONICET-Universidad de Buenos Aires, Argentina
}

Resumen: Fichte y Deleuze se interpelan recíprocamente cuando caracterizan -cada cual a su modo- al dogmatismo como el enemigo del pensamiento auténticamente filosófico. En este artículo, reconstruiremos y compararemos tales caracterizaciones para, a partir de ellas, interrogar a ambas ontologías y contemplarlas bajo una nueva luz. En Deleuze, se estudiará de qué manera el Yo al cual ubica -en todas sus dimensionesen el plano empírico puede hacer afirmaciones no-dogmáticas acerca de los planos que son su condición trascendental: lo intensivo y lo virtual. En Fichte, se refutará la interpretación de la célebre fórmula Yo = Yo como una forma de la identidad para sostener su comprensión como una inmanencia que produce eternamente la multiplicidad de lo real.

Palabras clave: dogmatismo, Deleuze, Fichte, inmanencia, Yo=Yo.

Abstract: Fichte and Deleuze question each other as they characterize -each in his own fashion- the dogmatism as the enemy of the authentic philosophical thinking. In this paper, we will reconstruct and compare such characterizations in order to examine both ontologies and consider them under a new light. In Deleuze, we will study how the I -all of whose dimensions are placed in the empirical field- can affirm something in a non-dogmatic way about the fields that are its transcendental condition: the intensive and the virtual. In Fichte, we will reject the interpretation of the famous sentence $\mathrm{I}=\mathrm{I}$ as a form of identity, in order to understand it as an immanence that produces eternally the multiplicity of the real.

Keywords: Dogmatism, Deleuze, Fichte, Immanence, I=I. 
$\mathrm{D}$ espertarse del sueño dogmático ha sido, desde Kant, una de las ambiciones de la filosofía (Kant, 1999: 29). Sin embargo, aspirar a la vigilia absoluta y alcanzarla son dos cuestiones bien diversas. Interrogar a Johann Fichte (1862-1814) y a Gilles Deleuze (1925-1995) acerca de la medida en que sus filosofías escapan efectivamente al dogmatismo nos permitirá a la vez vincular dos ontologías en apariencia contrapuestas y alcanzar importantes avances teóricos en ambos campos de estudio. La interrogación es absolutamente pertinente, ya que Fichte ha explícitamente colocado al dogmatismo como el gran rival de la filosofía, mientras que Deleuze ha hecho de la imagen dogmática del pensamiento la característica de lo no-filosófico. Por otra parte, ambos se consideran filósofos de la inmanencia, y ponen al dogmatismo y a la trascendencia en implicación recíproca. Así, en esta encrucijada se juega el destino de la inmanencia, y lo que está puesto en última instancia en cuestión es el nudo de los pensamientos de Fichte y Deleuze.

La filosofía comparada encontrará de esta manera un campo fértil para su labor. En efecto, si bien Deleuze toma como prius ontológico a la diferencia y Fichte retorna por el contrario una y otra vez sobre la unidad como requisito indispensable, de lo que se trata es de abordar el sentido último de la unidad en Fichte y la diferencia en Deleuze. Por una parte, mostraremos que la unidad fichteana no es incompatible con la diferencia en la medida en que es una actividad que nunca es idéntica a sí misma: no es la quietud del ser; así, no postula propiamente un fundamento (quieto) sino una actividad de fundación. Por otra parte, la diferencia deleuziana no implica un mero fluir indefinido del río de la vida, sino que da lugar a determinaciones e individuaciones. El desfondamiento no es incompatible con la fundación o el establecimiento de una tierra donde la vida sea posible. 


\section{El dogmatismo de acuerdo a Fichte}

Fichte identifica con claridad al rival de su filosofía: el dogmatismo. ${ }^{1}$ La caracterización fichteana de dogmatismo es específica, y no debe ser confundida con otras concepciones del mismo. ${ }^{2}$ Para Kant, por ejemplo, el dogmatismo consistía en tomar como punto de partida al entendimiento vacío (la pretensión de progresar únicamente por conceptos no sometidos a crítica), y lo contraponía al empirismo que partía de la sensibilidad ciega (Kant, 2009: 542). ${ }^{3}$ En cambio, bajo el rótulo común de dogmatismo, Fichte engloba ambos brazos de la antinomia kantiana, y también al propio Kant (en tanto su sistema da un lugar a la cosa en si). Podríamos en consecuencia distinguir tres fases del dogmatismo según Fichte: 1) el dogmatismo empirista que parte de la mera sensibilidad, ${ }^{4}$

${ }^{1}$ Debo la atención sobre el rol crucial del dogmatismo en la construcción del pensamiento de Fichte a Mariano Gaudio, quien en su tesis doctoral traza los caminos que ligan dogmatismo científico y despotismo político (Gaudio, 2016). ${ }^{2}$ Ferrater Mora indica en su diccionario tres sentidos de dogmatismo: 1) el realismo ingenuo, "que admite la posibilidad de conocer las cosas en su ser verdadero (o en sí)", 2) "la confianza absoluta en un órgano determinado de conocimiento, principalmente la razón", 3) "la completa sumisión sin examen personal a unos principios o a la autoridad que se nos impone" (1994: 929).

3 "[El dogmatismo es] la pretensión de progresar únicamente con un conocimiento puro por conceptos (el [conocimiento] filosófico), de acuerdo con principios como los que la razón tiene en uso desde hace tiempo, sin investigar la manera y el derecho con que ha llegado a ellos" (Kant, 2009: 35; Cfr. también 1999: 57). Para Hegel, consistía en afirmar en forma unilateral y fija una de las determinaciones contrapuestas del entendimiento (1970: 98-99).

${ }^{4}$ En la Primera introducción a la Doctrina de la ciencia encontramos las definiciones más explícitas en este sentido. Allí, Fichte define el dogmatismo como el intento de pensar la cosa con abstracción de la inteligencia cuando, según él, en la experiencia cosa e inteligencia van unidas. La cosa se transforma en cosa en sí (Cfr. Fichte, 1970a: 188). 
2) el dogmatismo racionalista que parte del mero entendimiento ${ }^{5}$ y 3) el dogmatismo crítico de Kant que postula la cosa en sí sin comprenderla (Fichte, 1994: 14). ${ }^{6}$ Los tres son para Fichte puntos de vista dogmáticos, en tanto aceptan una cosa (cosa empírica, intelecto como cosa, cosa en sí) independiente de la actividad (la actividad de la inteligencia, que "no es un ser, sino un actuar" (Fichte, 1970b: 225). ${ }^{7}$ Toda afirmación sobre ella no será más que mera fantasía, ilusión, y por tanto "inservible como fundamento explicativo de otra cosa” (Fichte, 1970a: 41). ${ }^{8}$

Empirismo, racionalismo y escepticismo9 son impugnados así en forma simultánea. La crítica del dogmatismo, en sus tres versiones, implica en breve que no podemos afirmar o aceptar nada absolutamente externo, independiente de nosotros. La solución fichteana es célebre: toda afirmación debe partir del Yo. Este Yo no puede ser el Yo empírico (lo cual implicaría un realismo ingenuo o dogmatismo empirista) sino un Yo absoluto, que en su actividad abraza y produce al Yo empírico -y a todo lo que el sentido común afirma como existente por sí-. Pero en realidad y en última instancia el Yo absoluto no puede ser otra cosa que el Yo empírico o finito que piensa o afirma algo determinado (por ejemplo, una Doctrina de la ciencia). Esta es la verdad de la famosa (y tan malinterpreta-

5 "[El dogmático dice] que los conceptos yacen en mi como algo primero y dado [fertiges]" (Fichte, 1994: 214).

${ }^{6} \mathrm{Si}$ bien en la primera introducción Kant "bien entendido" no sería dogmático, salvo para algunas interpretaciones sesgadas (Fichte, 1970a: 40), en la Nova methodo lo alineará explícitamente en esa perspectiva.

7 "El yo mismo no es nada más que un actuar sobre sí mismo" (Fichte, 1994b: 103). En pie de página de esa misma introducción al Fundamento del derecho natural, rechaza como absurdas las acusaciones contra la Doctrina de la ciencia como si "pusiera como fundamento de la filosofía un Yo en tanto sustrato existente sin la intervención del Yo (un Yo como cosa en sí)" (1994b: 103). Volveremos sobre las implicancias de este punto neurálgico del pensamiento de Fichte. ${ }^{8}$ Sobre la cosa en sí como invención o fantasía, cfr. Fichte (1970a: 36-37).

${ }^{9}$ En tanto resistencia a abordar la cosa en sí. 
da) fórmula "Yo $=$ Yo".${ }^{10}$ Si hay una escisión, un abismo entre el Yo finito y el Yo absoluto, mi Yo queda supeditado a una lógica extrínseca (aunque lo llame Yo). ${ }^{11}$ Ese abismo separaría el principio del actuar y el actuante; las leyes del actuar se le impondrían así desde afuera al Yo finito, que perdería de esa manera su libertad. ${ }^{12}$

${ }^{10} \mathrm{La}$ interpretación corriente (y errónea, desde mi punto de vista) enfatiza en la identidad. Esta puede interpretarse negativamente, como lo hace por ejemplo la "filosofía francesa de la diferencia" según Descombes (1988: 185), o positivamente, como Hegel en su célebre texto Diferencia entre los sistemas de filosofia de Fichte y Schelling, donde le reprocha a Fichte la autonomía entre el primer principio y el segundo, que inserta una oposición irreconciliable en el corazón de la identidad originaria que se trata de reivindicar (Hegel, 2010: 42-43). Sin embargo, la fórmula Yo = Yo no remite al principio de identidad, que para Fichte vacío y abstracto, meramente hipotético (si A se da entonces debe darse A, pero nada me garantiza que A se de efectivamente). Yo = Yo es por el contrario un principio concreto, categórico, con el contenido apodíctico del Yo que se da necesariamente; Fichte parte del principio de identidad como argumento retórico, pedagógico, suponiendo que nadie puede objetar ese "hecho cualquiera de la conciencia empírica" (Fichte, 1975: 14). Fichte inicia con ese argumento el movimiento de poner en duda la apodicticidad del principio de identidad, que encontrará su primer gran desarrollo en Hegel y será un rasgo distintivo de la filosofía francesa contemporánea.

${ }^{11}$ Esa lógica extrínseca es, en el artículo de Hegel citado en la nota anterior, el segundo principio o la oposición de un no-Yo al Yo. Pero también podría pensarse en una trascendencia de orden divino. En ese sentido, sería lo mismo Yo que Dios: "la sustitución del punto de vista de «Dios" por el punto de vista del "Yo» tiene mucha menos importancia que la que suele atribuírsele" (Deleuze, 1968: 117). Esto Fichte lo reconoce de alguna manera en su segunda etapa (después de 1800 y la polémica del ateísmo) cuando insiste en la identidad entre Dios y el saber. "No hay ningún abismo o separación entre Dios y el Saber" (Fichte, 1845: 450). Las interpretaciones continuistas de la filosofía de Fichte hacen eje en la poca importancia de la sustitución del punto de vista de Dios al del punto de vista del Yo. Hegel objetaría que el punto de vista del absoluto, lejos de implicar trascendencia de Dios respecto al Yo es la única posibilidad de recobrar la identidad originaria (o inmanencia plena).

12 "El dogmatismo parte de la falta de libertad y finaliza también con ella. Las representaciones son para él productos de las cosas, y la inteligencia o el sujeto 
Pero el principio de la Doctrina de la ciencia es la libertad del Yo. La cual sólo es posible en tanto se identifica con el Yo absoluto. El Yo se pone sus propios límites, y así la actividad es la fuente de las determinaciones de la realidad efectiva.

La refutación completa del dogmatismo se identifica de esta manera con la Doctrina de la ciencia misma, y la dificultad de la tarea se plasma en la secuencia de exposiciones de la misma a la que Fichte le dedicó toda su vida, y que hubieran sin duda continuado si no nos lo arrebataba la muerte, buscando una inalcanzable Doctrina de la ciencia que se identificara con la Doctrina de la ciencia.

\section{Spinoza en disputa}

Fichte incluye entre los dogmáticos a Spinoza, quien constituye uno de los pilares del sistema filosófico de Deleuze. ${ }^{13}$ Por lo tanto, es pertinente indagar en qué medida las críticas de Fichte a Spino$\mathrm{za}$, y en particular las vinculadas con el dogmatismo, impactan en la ontología de Deleuze, la interpelan, y obligan a profundizar en sus conceptos fundamentales. ${ }^{14}$

El punto más álgido de la polémica es la cuestión de la inmanencia: mientras ésta es el rasgo característico de la filosofía de Spinoza de acuerdo a Deleuze (y el motivo principal por el cual lo

son para él meramente pasivos" (Fichte, 1994a: 15).

13 "Spinoza es el Cristo de los filósofos [...] Mostró, estableció, pensó el «mejor» plan de inmanencia, es decir el más puro, el que no se somete a lo trascendente ni reestablece una trascendencia" (Deleuze y Guattari, 1991: 59). "Existe una única proposición ontológica: el Ser es unívoco [...] [Spinoza] en lugar de pensar al ser unívoco como neutro o indiferente, hace de él el objeto de una afirmación pura. El ser unívoco se confunde con la sustancia única, universal e infinita" (Deleuze, 1968: 52, 59).

${ }^{14}$ Para la relación directa entre Fichte y Spinoza, sin mediación a través de Deleuze, cfr. Solé (2013), Ivaldo (1992) y Hammacher (1992). 
reivindica), ${ }^{15}$ para Fichte el autor de la Etica demostrada según el orden geométrico es un filósofo de la trascendencia, y justamente por ese motivo su sistema es el dogmatismo más consecuente:

En el sistema crítico [el de Fichte] la cosa es aquello que es puesto en el Yo; en el sistema dogmático, [la cosa] es aquello en que el mismo Yo es puesto: el criticismo es por tanto inmanente, porque lo pone todo en el Yo; el dogmatismo es trascendente, porque incluso sobrepasa al Yo. El spinozismo es el producto más consecuente del dogmatismo, en la medida en que este puede ser consecuente (Fichte, 1975: 32; el subrayado es mío).

Para Fichte, mientras la sustancia spinozista trasciende a los modos, su Doctrina de la ciencia ("el sistema crítico") es el sistema de la inmanencia, y la única forma propia de la inmanencia: dado que soy Yo el que piensa, la única manera de no postular algo trascendente es que todo esté puesto en el Yo. En cambio en el dogmatismo el Yo es puesto en la cosa, que lo trasciende. En Spinoza, el Yo es inmanente a Dios, pero la recíproca no se cumple: Dios no es inmanente al Yo. Por lo tanto, la inmanencia no es completa. ${ }^{16}$ Los modos están, de acuerdo a la interpretación de Fichte, fuera de la sustancia, que es otra cosa que ellos. Así, la inmanencia

15 "Spinoza sabía plenamente que la inmanencia era inmanente a sí misma [...] por eso, era el príncipe de los filósofos" (Deleuze y Guattari, 1991: 49). Es el príncipe de los filósofos, en la medida en que era el sistema más acabado de la inmanencia, es decir, que no sólo la postulaba, sino que la haría "inmanente a sí misma” y no a algo trascendente, y objeto de una afirmación pura, y no un ser neutro.

${ }^{16}$ En su segunda etapa Fichte renunciará a esa "inmanencia completa": "Jesús no era Dios, porque no había ningún Jesús autónomo; Dios era en cambio Jesús, y aparecía como Jesús" (Fichte, 1845: 573). Recurriremos en varias oportunidades a este texto del segundo período, Introducción a la vida bienaventurada, que Deleuze destaca en "La inmanencia: una vida..." (Deleuze, 2003: 361). El lector debe considerar estas referencias con la debida precaución, en tanto para 
se quiebra: el modo trasciende a la sustancia, que es aquello por lo cual los modos son. Por otra parte, la sustancia de Spinoza es una cosa. No, por supuesto, una mera cosa, sino una cosa en sí, y una cosa en sí eminente en tanto permite deducir el dogmatismo más consecuente. Es consecuente en tanto, por una parte, afirma la sustancia sin ambages, la postula como primer principio y logra deducir de ella el sistema completo de la realidad (es decir, Dios es efectivamente causa inmanente del mundo). Pero esa sustancia como primer principio está postulada dogmáticamente, en tanto afirmamos algo acerca de lo que nos trasciende.

Lo cual nos impide tener un fundamento, es decir, derecho pleno a afirmarlo: “¿qué es, pues lo que contiene a su vez el fundamento de la necesidad de esta sustancia?" (Fichte, 1975: 32). Estamos aquí ante el segundo punto clave de la argumentación de Fichte: lo propio del dogmatismo no es sólo afirmar la cosa en sí, sino afirmarla sin fundamento. ${ }^{17} \mathrm{El}$ motivo por el cual el principio tiene que ser una actividad y no un estado (un ser, una cosa) es, en última instancia, que el ser fijo no permite fundar lo que se trata de fundar, y la actividad sí: "El problema de buscar el fundamento de una cosa contingente significa esto: mostrar otra cosa por cuya naturaleza se deje comprender por qué lo fundado tiene, entre la múltiples determinaciones que podrían convenirle, justamente aquellas que tiene" (Fichte, 1970a: 7). El dogmatismo busca el fundamento en la cosa en sí, mientras que la doctrina de la Ciencia lo busca en la inteligencia (8). Pero el dogmatismo "vulgar" fracasa, justamente, porque la cosa en sí no logra fundar lo que aspira a fundar. Spinoza, en cambio, como el dogmático más consecuente,

muchos intérpretes la discontinuidad entre el período de Jena y el posterior a 1800 es radical.

${ }^{17}$ Ese era también el punto de Kant: cuando distinguía el proceder dogmático (ciencia estrictamente demostrativa a partir de principios seguros) y el dogmatismo (partir de principios sin derecho) (Kant, 2009: 35). 
logra fundar la experiencia, pero no logra fundar el principio del cual la experiencia se deduce (la sustancia). El principio (Grundsatze) debe ser él mismo fundado. Escapar al dogmatismo implica entonces dar cuenta del fundamento o la fundación.

La crítica a Spinoza tiene implicancias para la comprensión de la Doctrina de la ciencia, como se observa al tener en cuenta cómo traduce Fichte el problema del dogmatismo spinozista en su propia terminología:

[Para Spinoza] el Yo (lo que él llama su Yo, o lo que yo llamo mi Yo) no es simplemente porque es, sino porque alguna otra cosa es [...] Un tal "fuera del Yo" sería igualmente un Yo, del que serían modificaciones el Yo puesto (por ejemplo: $m i$ Yo) y todos los Yo que puedan ser puestos. Separa la conciencia pura y la conciencia empirica. Pone la primera en Dios, que nunca es consciente de sí [...]; pone la segunda en las modificaciones particulares de la divinidad (Fichte, 1975: 19-20).

La sustancia de Spinoza sería el Yo absoluto o puro, mientras los modos serían Yoes finitos o empíricos: la traducción es ciertamente curiosa (dado que Spinoza no llama "Yo" a ningún concepto técnico y, por tanto el "lo que él llama su Yo" adquiere una connotación irónica) pero sumamente clarificadora, en tanto revela que la fórmula Yo $=$ Yo expresa la inmanencia recíproca de sustancia y modos (sustancia $=$ modos). El "=" no indica por lo tanto propiamente identidad sino inmanencia. Pero esta inmanencia fichteana debe cumplir el requisito que le exige a Spinoza: ser capaz de fundar, tener derecho a afirmar lo que afirma. Yo = Yo, como actividad propia del Yo absoluto debe permitir entonces fundar la experiencia y al Yo empírico que la experimenta como algo real, pero también dar cuenta de su propio fundamento, es decir, del derecho que tenemos de afirmar el Yo absoluto como principio. Volveremos en 
el apartado 5 sobre este punto clave de la ontología de Fichte, y todos los problemas que encierra.

\section{Deleuze: inmanencia y dogmatismo}

Deleuze no ignora el argumento de Fichte según el cual la sustancia de Spinoza podría trascender a los modos. ${ }^{18}$ De hecho, en medio de una actitud en general apologética de la doctrina de Spinoza, esa es una de las pocas críticas que le realiza: "La sustancia spinozista aparece como independiente de los modos, y los modos dependen de la sustancia, pero como de otra cosa" (Deleuze, 1968: 59). ${ }^{19}$ La crítica es la misma (los modos están separados de la sustancia) pero la solución es, al menos a primera vista, diversa. Dice Deleuze: "Sería necesario que la sustancia se dijera ella misma de los modos, y solamente de los modos. Que la identidad no es primera, que no existe como principio, sino como segundo principio, como principio devenido; que gira en torno a lo Diferente" (Deleuze, 1968: 59). La sustancia se dice solamente de los modos, pero los modos ellos mismos no se resuelven en una unidad, sino que giran en torno a lo diferente. Lo Diferente aparece así como el principio ontológico fundamental de la ontología de Deleuze. Esta diferencia será caracterizada en términos más precisos como multiplicidad (cuyas características detallamos más abajo).

\footnotetext{
${ }^{18}$ No se puede afirmar que la tome de él, pero sí que Deleuze tenía presente desde muy temprano la crítica de Fichte a la cosa en sí, tal como testimonia una carta de 1964: "No hay dudas de que la teoría de las cosas en sí tiene dos aspectos: como sustrato de los fenómenos, como fundamento de la voluntad -fuera de nosotros, y en nosotros [...] El problema se plantea efectivamente en el kantismo, pero no es resuelto por Kant mismo. Sí lo resuelven, por una parte Fichte, y por la otra Schopenhauer" (Deleuze, 2015a: 17-18).

${ }^{19}$ Esta crítica dista de ser reiterada y constante. En general, atribuye a Spinoza una inmanencia completa: "En Spinoza, la inmanencia no es $a$ la sustancia, sino que la sustancia y los modos están en la inmanencia" (Deleuze, 2003: 360).
} 
Que Deleuze establezca la multiplicidad como principio es, en apariencia, el principal punto de inconmensurabilidad con Fichte, dado que para el filósofo idealista la unidad del absoluto es un requisito indispensable para la Doctrina de la ciencia: "Una ciencia debe ser algo Uno, un Todo", dice en Sobre el concepto de la Doctrina de la ciencia (Fichte, 1963: 14-15), y precisa en la Ética: "nuestra actividad no es ninguna multiplicidad, sino absoluta identidad pura" (Fichte, 1977: 98). A pesar de lo taxativo de estas afirmaciones, esta inconmensurabilidad es relativa porque, por un lado, como veremos en el apartado 5 de este trabajo, la Unidad fichteana no es fija y quieta, sino una actividad perpetua de autolimitarse, y por tanto no es absolutamente hostil a la multiplicidad; ${ }^{20}$ por otra parte, la multiplicidad deleuziana se enmarca en una filosofía de la univocidad ("el ser es el mismo para todas sus modalidades"; Deleuze, 1968: 53), y en ese aspecto es Una. Quizás tengan razón Deleuze y Guattari cuando dicen que la fórmula mágica que todos buscamos es pluralismo $=$ monismo $^{21}$

A primera vista, sin embargo, Deleuze cumpliría todos los requisitos para ser un dogmático en sentido fichteano. Lo Diferente como principio implica que cada modo es distinto de los otros, que por tanto mi Yo (lo que Deleuze llamaría su Yo) no contiene ni puede contener a los otros. Pero entonces mi Yo es porque otra cosa es: el resto de lo diferente, la multiplicidad, el hormiguear de los modos que constituyen la inmanencia. Deleuze se parece aquí al dogmático que "niega totalmente la independencia del yo, sobre el cual construye el idealista, y hace de él solamente un producto de las cosas, un accidente del universo" (Fichte, 1970a: 41). Lo único

${ }^{20}$ De hecho, Fichte admite una multiplicidad de la experiencia infinita, de la cual no puede haber conocimiento más que a posteriori (1845: 459).

21 "Llegar a la fórmula mágica que todos buscamos: PLURALSIMO = MONISMO" (Deleuze, 1980: 31). Sobre Deleuze como solución a la fórmula "Fichte + Spinoza”, parafraseando esta cita (Cfr. Amrine, 2015). 
que habría logrado Deleuze al reducir la sustancia a los modos es resignar lo principal que Fichte defendía de Spinoza, y que lo hacía a sus ojos un dogmático al menos consecuente: la unidad. La unidad de la sustancia es resignada en nombre de la multiplicidad de los modos. Pero si esto tenía como fin librarse de la trascendencia dogmática que la sustancia implicaba (punto en el cual Deleuze y Fichte están de acuerdo), pareciera que la trascendencia es reinstaurada entre los modos.

Sin embargo, esta apariencia sólo tendría validez si Deleuze estuviera desplazándose hacia un empirismo vulgar. Pero Deleuze impugna tomar como punto de partida "el elemento de la sensación (empirismo simple)" (Deleuze, 2003: 359) al cual, por otra parte, considera, como Fichte, un dogmatismo. ${ }^{22}$ Es cierto que Deleuze se define en numerosas ocasiones a sí mismo como empirista $^{23}$, pero su empirismo es trascendental, un "empirismo superior lo vincula con la inspiración antidogmática que anima al poskantismo" (Krtolica, 2015: 22). ${ }^{24}$

La idea de un empirismo trascendental, sostiene por una parte que hay una diferencia de naturaleza entre lo empírico y lo trascendental; por otra parte, supone que lo trascendental es él mismo experiencia, experimentación; finalmente, plantea una inmanencia completa entre los dos (Deleuze, 2015a: 89).

${ }^{22}$ En su libro de 1963 sobre Kant, Deleuze señala que "el empirismo de Hume tenía un desenlace similar" al del dogmatismo, debiendo recurrir a la armonía preestablecida para explicar la correspondencia entre el orden de las Ideas y el orden de las cosas (Deleuze, 1963: 22).

${ }^{23}$ Hay que tener también en cuenta que su primer libro está dedicado a Hume. ${ }^{24}$ Krtolica señala puntualmente el poskantismo de Schelling, pero se puede extender a Fichte, quien es el que propiamente tiene una inspiración antidogmáti$\mathrm{ca}$, que en todo caso Schelling retoma en sus escritos tempranos. Curiosamente, esta afirmación no impide que Krtolica realice, unas páginas más adelante, una reconstrucción bastante dogmática del empirismo deleuziano (2015: 33-34). 
Esta forma específicamente deleuziana del empirismo toma como principio la multiplicidad o diferencia, pero ésta no debe confundirse con lo múltiple del empirismo vulgar o del dogmatismo empirista. Es indispensable por tanto establecer una distinción entre tres tipos de diferencia, cuya confusión explica gran parte de los malentendidos que giran en torno a la ontología de Deleuze: las diferencias virtuales (o ideales), las diferencias intensivas y las diferencias empíricas (sólo las dos primeras son multiplicidades ${ }^{25}$ mientras las últimas son meramente múltiples).

Lo múltiple o los sistemas de diferencias empíricas tienen por su parte un doble aspecto: extensivo (o conjunto de partes) y cualitativo (o relaciones reales o espacio-temporales). El caso más elocuente es el de los sistemas biológicos, donde cada especie es definida por ciertas partes singulares (como es el caso paradigmático de los órganos reproductivos de las plantas en Lineo) y además por ciertas relaciones especificas (como estructura o composición de sus cuerpos), que según Deleuze no serían modos alternativos de clasificación natural sino complementarios. ${ }^{26}$ En el caso del sistema del Yo, Deleuze señala la relación recíproca entre el Yo como cualidad (Yo pienso como especificidad del hombre) y el Yo como extensión ("los puntos notables representados por las diversas facultades que entran en la comprensión del Yo"; Deleuze, 1968: 330). ${ }^{27}$ Desde la perspectiva deleuziana, el Yo no da cuenta de

25 "Las Ideas son multiplicidades virtuales, problemáticas o "perplejas [perplexes, formado con el prefijo "per" -en torno a- y "plex", pliegue], hechas de relaciones entre elementos diferenciales. Las intensidades son multiplicidades implicadas, «implejos» [implexes, formado con el prefijo "in" -dentro de-], hechas de relaciones entre elementos asimétricos, que dirigen el curso de actualización de las ideas" (Deleuze, 1968: 315).

${ }^{26}$ Deleuze se basa en la concepción de la historia natural como correlación partes-estructura planteada por Foucault (1966: 144-458).

${ }^{27} \mathrm{La}$ multiplicidad de las facultades ha sido reconocida y tematizada por Fichte, y por todo el idealismo alemán. Deleuze afirma que no hay armonía posible 
sí mismo, no encuentra en sí su fundamento. Por el contrario, está esencialmente fisurado, disuelto, desgarrado entre su aspecto cuantitativo y cualitativo (Deleuze, 1968: 332). El argumento fichteano se invierte, el Yo no ofrece certeza, la intuición intelectual no garantiza unidad, porque el Yo que intuye y el Yo intuido no son el mismo, sino que son una cualidad y una cantidad cuyo enlace sólo puede ser afirmado dogmáticamente. La unidad del Yo es una cuestión de hecho, pero no de derecho.

Deleuze no niega que haya individuos y que estos se expresen como yoes, ni que estos yoes piensen; habla explícitamente de "la individualidad concreta del pensador" (1968: 333). Sólo indica que para dar cuenta de ellos tenemos que remitirnos a las multiplicidades que los constituyen y crean. La cuestión de derecho sólo puede resolverse en el plano trascendental ${ }^{2}$, donde se revela que ese múltiple doble (cualidad-extensión) que constituye al Yo está fundado por multiplicidades virtuales e intensivas.

Esta diferenciación [differenciation] fundamental (cualidad-extensión) sólo puede encontrar su razón en el [...] orden implicado de la intensidad [...] Únicamente el estudio trascendental puede descubrir que la intensidad sigue implicada en sí misma y continúa

entre ellas, que no se resuelven en una identidad, y que no tienen una relación armónica entre sí. No encuentran concordia en un sentido común (Deleuze, 1968: 174). Su unidad es meramente empírica, y es postulada en forma dogmática y no trascendental. El Yo pienso no es así menos empírico que la conciencia vulgar o la experiencia corriente.

${ }^{28}$ Lapoujade señala que la filosofía de Deleuze no es ajena a cuestión de derecho como juicio de lo bien fundado de una pretensión: "un hecho debe ser concebido como una pretensión, una exigencia o una reivindicación y la pregunta quid juris? tiene como función justamente juzgar lo bien fundado de la pretensión [...] cuestión que está ya en el corazón de Diferencia y repetición"(2015: 24-25). 
envolviendo la diferencia, en el momento en el que se reflexiona en la extensión y la cualidad que crea (Deleuze, 1968: 308-309). ${ }^{29}$

Este pasaje es clave. En primer lugar, explicita que la razón del Yo (en tanto diferenciación cualidad-extensión) está en el orden intensivo. La intensidad es así un principio de razón, que estructura y determina el sistema psíquico. Yo pienso, efectivamente, pero yo que pienso sólo soy este pensador concreto en la medida en que soy un individuo cuya razón está en el orden intensivo: "el mundo implicado de las cantidades intensivas constituyen la individualidad concreta del pensador" (Deleuze, 1968: 333). La intensidad es la dimensión que sostiene las partes discretas. Y no sólo las sostiene (en tanto condición) sino que también las crea. La proclamada "independencia" del Yo no es más que una abstracción de su aspecto trascendental, que hace que la estructura yoica no sea propia, sino ajena, no esté en el Yo, sino en el Otro. ${ }^{30}$

Pero hay un segundo motivo por el cual el pasaje es mucho más relevante para la investigación propuesta en estas páginas: el carácter implicado de la intensidad. Esto es vertebral para el problema de la inmanencia. Porque cuando Deleuze dice que la intensidad se anula en la cualidad y la extensión que crea (1968: 309), pareciera haber una relación de transcendencia entre la intensidad y lo extensivo (si se anula trasciende a lo empírico). Pero no se anula absolutamente, sino que en el orden de la cualidad y la extensión el

${ }^{29}$ Eludimos el contrapunto con Bergson que Deleuze utiliza aquí, que nos llevaría a análisis que no son pertinentes en este contexto. El punto es el siguiente: la cualidad no es la razón de la cantidad, como Bergson da a entender por momentos, sino que ambas encuentran su razón en la intensidad (que sería la tesis esencial de Bergson, el bergsonismo bien entendido).

${ }^{30}$ Sobre el rol del Otro (Autrui) como expresión de un mundo posible, cfr. Deleuze (1968: 333-335, 360). Respecto la relación entre la otredad deleuziana y la fichteana (en su desarrollo de la intersubjetividad y el rol constituyente del otro), cfr. Ferreyra (2016a). 
ejercicio empírico de la sensibilidad puede captar la intensidad, en tanto la intensidad está implicada en lo empírico. Es decir, podemos captar la intensidad a través del ejercicio empírico de la sensibilidad en tanto la intensidad está envuelta en lo empírico. De esa manera, la intensidad no es una cosa en sí, una trascendencia y, por lo tanto, no se afirma dogmáticamente.

Sin embargo, lo que puede ser captado empíricamente es meramente una implicación segunda, en la cual la intensidad aparece en su grado más bajo, "sólo lo mínimo necesario que hace falta para "explicarla»" (309); la intensidades implicadas, envueltas, crean explicándose o desenvolviéndose, pero lo hacen sólo en forma baja. Más que la razón de la extensión, parecen simples anomalías. Por eso es tan importante el orden de implicación primero en el cual "la intensidad está implicada en sí misma, a la vez envolvente y envuelta” (Deleuze, 1968: 309). Dado que no hay trascendencia entre los ordenes de implicación (es la misma intensidad, la misma implicación) también podemos afirmar sin dogmatismo lo más alto, la auténtica razón de lo sensible, la intensidad que crea la extensión diferenciándose. Aunque no podamos experimentarlo de manera sensible.

Al mismo tiempo, debemos recordar que la intensidad no es una, sino múltiple. No hay una intensidad, sino una multiplicidad de intensidades, que no tienen una relación extrínseca (partes extra partes) como lo múltiple de la extensión, sino intrínseca: "las intensidades envuelven otras intensidades, están envueltas por otras y comunican con todas" (Deleuze, 1968: 331). Envolvimiento mutuo de las intensidades, que da cuenta de la complejidad de las unidades empíricas, ya que éstas explican una multiplicidad de intensidades: biológicas, sociales, físicas, lingüísticas, etc. Entre todas constituyen la individualidad del sujeto concreto, empírico, que puede pensar, hablar, moverse, contar con el latido de su corazón y su respiración, formar parte de una sociedad que evita que lo 
destruya la ley del más fuerte, etc. Esta multiplicidad de modos de existencia estaría dispersa, fisurada, atomizada, si no fuera por su relación de envolvimiento con su razón intensiva.

Ahora bien, las intensidades dan cuenta de la individuación, pero no de la determinación de los individuos. ¿Por qué un Yo pienso, por qué las facultades? ¿Por qué una especie, por qué tales partes? ¿Por qué este sistema circulatorio, por qué el corazón? La razón suficiente de las cualidades y extensiones se encuentra en el concepto de Idea. Las Ideas deleuzianas son relaciones entre elementos diferenciales, infinitamente pequeños $(d y, d x)$, cuya diferenciación consiste en la producción de singularidades. Lo infinitamente pequeño (que sólo existe en relación), sus relaciones y las singularidades constituyen la multiplicidad o diferencia virtual. Las relaciones diferenciales se diferencian en lo empírico como relaciones actuales (cualidades) y las singularidades como partes notables (extensiones).

Una vez más, el problema del dogmatismo exige dar cuenta de la manera en que la inmanencia no se encuentra quebrada por el recurso a esta razón ideal. Para eso es necesario concebir el plano virtual como un espacio topológico. ${ }^{31}$ Las multiplicidades virtuales no son entidades discretas y no existen sobre un espacio topológico, sino que son ellas mismas ese espacio. Pero tampoco lo múltiple es trascendente, ya que el espacio euclidiano no trasciende al topológico sino que nace de él: "En esta versión metafísica, los espacios métricos nacen literalmente de los no-métricos" (DeLanda, 2010: 130).

¿Cómo se vincula la multiplicidad virtual con la multiplicidad intensiva? Bajo la forma del plegado. En el espacio topológico, el estiramiento y el plegado permiten pensar la univocidad de todos los cuerpos que, en las otras geometrías, aparecen como distintos

${ }^{31}$ Sobre las características del espacio topológico y su distinción con el euclideano, cfr. DeLanda (2010: 118-122). 
y recíprocamente trascendentes. La diferencia de intensidad no es una división o escisión respecto al espacio topológico, sino su pliegue. ${ }^{32}$ Las diferencias intensivas son pliegues del campo de inmanencia. Ningún pliegue trasciende al otro: una misma tela para todos los pliegues en la cual se derrama. Lo virtual es la tela plegada por el pliegue, el océano donde se producen las olas, el espacio topológico que la intensidad pliega y estira, la dimensión última de la ontología deleuziana. La individualidad del pensador concreto se constituye como pliegue: "Pensar es plegar, pensar es curvar [...] Es la topología del pensamiento” (Deleuze, 2015b: 181). Así, "el lazo del pensamiento con la individuación es mucho más profundo que el que aparece con el Yo pienso" (Deleuze, 1968: 197). Gracias al concepto de intensidad como pliegue del campo topológico virtual-ideal, podemos dar cuenta de la inmanencia del plano virtual y el intensivo, y de las multiplicidades correspondientes entre sí (un pliegue no trasciende a otro pliegue porque son pliegues del mismo espacio, una Idea no trasciende a otra idea porque son diferenciaciones de la misma virtualidad, continuas y esencialmente relacionales). La ontología de Deleuze parece estar a resguardo del espíritu del dogmatismo que Fichte consideraba el peor enemigo del pensamiento auténticamente filosófico.

\section{El dogmatismo según Deleuze}

"El error del dogmatismo ha sido colmar lo que separa [...en ese sentido, hay todavía] demasiado dogmatismo en los poskantianos" (Deleuze, 1968: 221). ¿En qué consiste este "colmar" [combler] que sella según Deleuze el destino dogmático del poskantismo, y por tanto de Fichte? Siguiendo como hilo conductor las definicio-

${ }^{32}$ Si bien el concepto de pliegue es desarrollado con detalle por Deleuze recién el la década de los ochenta, en los libros de los sesenta lo podemos encontrar bajo las figuras de la implicación, explicación y complicación (Deleuze, 1968: 297). 
nes deleuzianas de dogmatismo dispersas en su obra, este acto de colmar tendría la forma de una armonía. "El dogmatismo afirmaba una armonía entre el sujeto y el objeto, invocando a Dios para garantizar esta armonía” (Deleuze, 1963: 98): ${ }^{33}$ Deleuze se refiere aquí al dogmatismo racionalista pre-crítico, donde Dios funcionaba como principio unificador, colmando la brecha entre sujeto y objeto. Los poskantianos, sin embargo, no son propiamente dogmáticos, sino demasiado dogmáticos, es decir, demasiado para su pretensión de eludir en forma radical todo atisbo de dogmatismo. Es indudable que para los poskantianos Dios ya no garantiza la armonía; la armonía no está preestablecida, no está dada. Tiene que ser, en cambio, conquistada: "Una furia de fundar, de conquistar, inspira a esta filosofía [alemana]" (Deleuze y Guattari, 1991: 100). Si para Fichte, la armonía consiste en "la coincidencia con nosotros mismos" (Fichte, 1994a: 106), ${ }^{34}$ entonces su conquista consistiría en realizar el Yo = Yo (que, es, por cierto, equivalente a Sujeto $=$ Objeto $).{ }^{35}$

En suma, según la perspectiva deleuziana, el dogmatismo de Fichte consistiría en garantizar la armonía entre el sujeto y el objeto bajo la forma del Yo = Yo (como fórmula de la coincidencia con nosotros mismos), sin invocar a Dios sino nuestra propia actividad: la conquista. Pero, esta conquista sólo sería propiamente dogmática si el "=" expresara una identidad, la identidad del Yo que forma parte de la imagen dogmática del pensamiento que Deleuze expone en el tercer capítulo de Diferencia y repetición. ${ }^{36} \mathrm{El}$ sentido

33 "En el racionalismo dogmático, la teoría del conocimiento se fundaba en la idea de una correspondencia entre el sujeto y el objeto, de un acuerdo entre el orden de las ideas y el orden de las cosas" (Deleuze, 1963: 22).

${ }^{34} \mathrm{La}$ cita exacta es: "La verdad es la coincidencia con nosotros mismos, armonía” (Fichte, 1994a: 106).

35 "El Yo no es en absoluto Sujeto sino Sujeto = Objeto" (Fichte, 1994a: 31).

${ }^{36}$ Cfr. el tercer postulado, "del reconocimiento": "Tal es el sentido del Cogito como comienzo: expresa la unidad de todas las facultades en el sujeto, y por lo 
del dogmatismo en Deleuze debe necesariamente comprenderse a la luz de su concepción de la imagen del pensamiento (que el autor de Diferencia y repetición combate por su carácter dogmático con el mismo fervor que Fichte combate el dogmatismo de su época, y por los mismos motivos: constituyen dos maneras incompatibles de hacer filosofía). La fórmula Yo = Yo implicaría, de acuerdo con esto, identidad y no inmanencia, y expresaría en forma acabada el pensamiento de Fichte. ${ }^{37}$ Es demasiado dogmático porque el "=" colma la separación entre el Yo y el Yo.

Al mismo tiempo, esta coincidencia expresaría la verdad: "La verdad es la coincidencia con nosotros mismos, armonía" (Fichte, 1994a: 106), lo cual confirmaría su carácter dogmático en sentido deleuziano: "de acuerdo a esta Imagen [dogmática], el pensamiento tiene afinidad con lo verdadero, posee formalmente lo verda-

tanto expresa la posibilidad de que todas las facultades se relacionen con una forma de objeto que refleja la identidad subjetiva, y le da un concepto filosófico al presupuesto del sentido común, es el sentido común que se ha vuelto filosófico. Tanto en Kant como en Descartes, la identidad del Yo [Moi] en el Yo [Je] pienso funda la concordancia de todas las facultades, y su acuerdo bajo la forma de un objeto que se supone como el Mismo" (Deleuze, 1968: 174). Teniendo en cuenta los desarrollos del apartado precedente, el dogmatismo consistiría en suponer la armonía entre los dos aspectos del Yo empírico (cuantitativo y cualitativo) que es lo que se trata de demostrar.

${ }^{37}$ Deleuze, como tantos otros, refiere a Fichte indirectamente a través de la fórmula Yo = Yo en varias ocasiones: "la forma del concepto como forma de identidad, que constituye tanto el en sí del representado [A es A] como el para sí del representante (Yo = Yo)" (Deleuze, 1968: 79); "hace falta que el contrato llegue hasta el final, que no se haga ya entre dos personas, sino entre sí mismo y sí mismo, en la misma persona, $I c h=I c h$ " (Deleuze y Guattari, 1980: 575). Sabe sin embargo que es una fórmula trillada, que debe ser pensada en forma más compleja: "Es asombroso porque quiere decir que el yo sólo se plantea como idéntico a sí oponiéndose a un no-yo. Es ciertamente no cartesiano, es la filosofía poskantiana. Es el yo = yo de Fichte: la autoposición del yo implica la posición del yo con un no-yo. El yo sólo se sitúa como yo por negación del no-yo" (Deleuze, 2011: 588). 
dero y desea materialmente lo verdadero" (Deleuze, 1968: 172). La única desventura del pensamiento es el error. Pero el error debe su existencia a la verdad, y es todo lo que la verdad rechaza como falso e ilusorio (todo lo que difiere del Yo, todo lo atribuible al no-Yo, desde esta perspectiva, tiene como sólo destino su disolución, su absorción en la verdad del Yo). Por su parte, la verdad es lo afín al pensamiento, por lo tanto el pensamiento volverá, tarde o temprano, al camino recto. Siempre de acuerdo con la imagen dogmática, el trabajo del filósofo es, en todo caso, disipar las ilusiones, las tentaciones al desvío. Purificar la verdad de todo error. Conquistar la inmanencia consiste entonces en erradicar lo erróneo, lo imperfecto, lo que escapa a la acción del Yo. La realización de la identidad Yo = Yo consistiría en esa erradicación, y por lo tanto el dogmatismo consistiría en ignorar que hay algo trascendental en las desventuras del pensamiento, algo necesariamente impuro en el saber, algo inherentemente estúpido en nuestra inteligencia. ${ }^{38}$ Para Deleuze, en cambio, no hay nada natural en el pensar y sólo se piensa coaccionado, forzado por la violencia de lo que fuerza a pensar. Lo que aparece como ajeno al pensador debe ser comprendido como intrínseco, dado que es lo que hace posible el pensamiento como movimiento aberrante, agrietando la supuesta identidad del Yo en favor de la inmanencia bajo el principio de la Diferencia, arrastrando a los dos aspectos empíricos del Yo (cuantitativo y cualitativo) a la condición trascendental de su génesis: las multiplicidades intensivas y virtuales que estudiamos en el apartado precedente.

${ }^{38}$ Respecto a esta cuestión en Deleuze, confrontar los desarrollos sobre la "bêti$s e$ " (estupidez) como bestialidad propiamente humana, y su contraposición a la figura del error (Deleuze, 1968: 194-198). Sobre el tema puede consultarse Ferreyra (2016b). 


\section{Fichte en el laberinto del dogmatismo}

Resumiendo: si Fichte fuera dogmático en sentido deleuziano, el Yo = Yo sería simple identidad, simple expresión de la armonía como verdad que expulsaría fuera de sí toda inadecuación en tanto error; así, el pensamiento estaría en afinidad con lo verdadero y sería una experiencia natural, ajena a toda violencia. Esta es la interpelación que Deleuze lanza al pensamiento fichteano.

Si tomamos el plan trazado por Fichte en Sobre el concepto de la Doctrina de la ciencia, la crítica parece aplicarse plenamente, ya que dicho plan exigía encontrar en el fundamento o principio fundamental (Grundsatz) un hilo de Ariadna a través de los laberintos del "error"; ${ }^{39}$ ese hilo que nos aparta de las desventuras del pensamiento es la unidad del fundamento: un fundamento único sostiene todo el sistema (Fichte, 1963: 13). El primer principio (Grundsatz), el Yo = Yo, parece en el Fundamento de toda la Doctrina de la ciencia el candidato natural para ocupar el rol de fundamento, único e idéntico, que garantiza la unidad del sistema, el buen sentido, el camino recto: lo que Deleuze llama la filosofía de la identidad, que su filosofía de la diferencia viene a destronar. Esa lectura se refuerza cuando se cree que esa fórmula se basa en

39 "Al menos una proposición tendría que ser cierta, la cual comunicara a las demás su certeza [...] todas ellas - tendrían certeza, y la misma certeza (...) Una tal proposición cierta previamente al enlace se llama proposición fundamental' (Fichte, 1963: 15-16). De esa manera, "el sistema del espíritu humano, cuya exposición ha de ser la doctrina de la ciencia, es absolutamente cierto e infalible; todo lo que está fundado en él es simplemente verdadero; él no yerra nunca, y lo que alguna vez ha sucedido o sucederá en un alma humana es verdadero" (46-47). Fichte nunca deja de considerar que el pensamiento recto conduce a la verdad, que el error es un desvío que puede y deber ser evitado: "El pensamiento científico en efecto separa la verdad del error que se le opone en todo respecto" (Fichte, 1845: 422). 
el principio de identidad $(\mathrm{A}=\mathrm{A}) .{ }^{40}$ El pensamiento encontraría su reposo, su quietud en la identidad del principio y quedaría a salvo de toda violencia. La filosofía depone sus armas, y la Doctrina de la ciencia se inscribe, desde esta perspectiva, en la imagen dogmática del pensamiento que Deleuze impugna.

Si muchos comentaristas insisten en leer así toda la Doctrina de la ciencia, ha sido por una parte por atenerse excesivamente al plan trazado en Sobre el concepto y, por otra parte, merced al efecto de la deslumbrante luz de la célebre fórmula Yo = Yo. Sin embargo, la convicción sobre la existencia de un camino recto que nos aparte del error, tan fuerte en 1794 se ve puesta en duda por el curso del trabajo de Fichte. Ya en 1796 comienza a trabajar sobre una nueva exposición de la Doctrina de la ciencia, que elaborará durante sus cursos hasta 1799 y que será conocida como la Doctrina de la ciencia nova methodo. ${ }^{41} \mathrm{La}$ aparición de los tres manuscritos de alumnos en el siglo XX generó, con toda razón, una revolución en los estudios sobre Fichte. Entre muchos aportes teóricos, allí desaparece el Yo = Yo como primer principio, y su lugar lo ocupa la intuición intelectual, la experiencia directa de la Thathandlung. Ahora bien, la Thathandlung implica una violencia del pensamiento natural. Por ello Fichte dice en la Nova methodo casi exactamente lo mismo que Deleuze acerca de la necesidad de una violencia que

${ }^{40}$ Sobre la inconveniencia de interpretar la filosofía de Fichte en términos de identidad, cfr. nota 10 .

41 "Fichte no nos dejó ningún manuscrito de la Doctrina de la ciencia nova methodo, cuyas clases impartió en Jena entre 1796 y 1799. Fichte había sin embargo comenzado esta Doctrina de la ciencia como «Versucht einer neuen Darstellung der Wissenshaftslehre» [...] cuya publicación se interrumpe por motivos desconocidos después del primer capítulo" (Fuchs, 1994: IX). Las Introducciones a la Doctrina de la ciencia anteceden ese texto interrumpido. La Doctrina de la ciencia nova methodo es el resultado de esas clases, de las cuales tenemos tres apuntes de alumnos. Nosotros utilizaremos el de Krause y el conservado en la biblioteca de Halle. 
fuerce a pensar: "Pienso realmente cuando me siento coaccionado a ello" (Fichte, 1994a: 217). Fichte desconfía del sentido común y el pensamiento natural (primer postulado de la imagen dogmática del pensamiento). El buen sentido está dominado por los fanáticos del mundo del revés. El pensamiento sólo puede ponerlo al derecho a través de un largo esfuerzo, cuya muestra patente es la elaboración y reelaboración de la Doctrina de la ciencia que Fichte llevará a cabo a lo largo de toda su vida.

Las sucesivas exposiciones de la Doctrina de la ciencia muestran de hecho que el pensamiento para Fichte no está dado, sino que debe ser conquistado, y, más aún, engendrado. En efecto, si bien la necesidad de una nueva exposición en la Primera introducción a la Doctrina de la ciencia es atribuida por Fichte a una coacción externa ("mi teoría no se entiende... me esforzaré por conseguir una mayor claridad), " ${ }^{42}$ dicha coacción es en realidad interna: la exposición de la Doctrina de la ciencia debe reformularse una y otra vez, porque existe una brecha constitutiva entre la Doctrina de la ciencia y su expresión (como ya insinuaba la primera línea del Fundamento de 1794: "El primer principio debe expresar aquella Thathandlung"; Fichte, 1975: 13). Si bien la letra empirica -la expresión- debe esforzarse incesantemente en expresar el espíritu trascendental, dicha letra está quieta y fija, mientras lo que debe ser expresado está por el contrario en actividad perpetua -la Thathandlug-; por lo tanto, la expresión no puede nunca encontrar su forma última. ${ }^{43} \mathrm{Y}$ esto

42 "Chanzas, desdenes y el universal testimonio de que se repugna esta teoría de todo corazón, como también de que no se la entiende. Por lo que respecta a esto último, quiero cargar yo solo con toda la culpa (...) Si es que puede darle gusto al lector entrar en la presente exposición, en la que me esforzaré por conseguir la mayor claridad" (Fichte, 1970a: 184).

${ }^{43}$ En "Sobre la diferencia entre el espíritu y la letra en la filosofía", señala Fichte precisamente el riesgo de la mera filosofía de fórmulas, de quedarse "sólo en la letra de ese principio [El Yo que se pone absolutamente] sin haber captado, no obstante, su espíritu" (Fichte, 1998: 152). 
por motivos fundamentales: la inadecuación constitutiva entre lo empírico y lo trascendental. El Yo nunca debe efectivamente devenir idéntico al Yo, so pena de aniquilación de la esencia activa del sistema. La clave está, entonces, no en una u otra exposición de la Doctrina de la ciencia, sino en su transformación continua.

Esta inadecuación marca que no hay una mera "identidad" en la inmanencia fichteana, sino también una diferencia. Esa diferencia entre lo empírico y lo trascendental, no es otra cosa que la diferencia entre el Yo absoluto y el Yo finito, y eso es lo que en realidad expresa la fórmula $Y o=Y o$. Es decir, que el principio no es el $\mathrm{Yo}^{1}$ ni el Yo ${ }^{2}$, sino el "=" -la X en el Fundamento de 1794- (1975: 15): "el pensamiento no debe partir desde los extremos, sino desde el punto medio" (Fichte, 1845: 510). El “=” es el verdadero principio de la filosofía que, lejos de colmar lo que separa, le impone movimientos infinitos, haciendo resquebrajar el pensar empírico que acontece en nuestras mentes orgánicas. Es una síntesis, sí, pero una síntesis que no unifica partes ya dadas, sino que desarrolla algo enteramente nuevo e inexistente con anterioridad (Fichte, 1994a: 189) ${ }^{44}$. La identidad que Deleuze le atribuye a tal síntesis no es más que la consecuencia de ver la Doctrina de la ciencia " $d u$ petit côtê". 45

${ }^{44}$ Esto es lo que Hegel no capta en el escrito de la diferencia cuando insiste, una y otra vez, en que Fichte parte de una escisión que nunca logra superar. Coherentemente con ello piensa que Yo $=$ Yo es una identidad pura sin límites ni fisuras, al menos por derecho ("El yo debe ser igual al yo", Hegel, 2010: 51) y que "la libertad es lo que supera en sí toda limitación y lo supremo del sistema fichteano" (63).

45 Parafraseamos a Deleuze cuando dice que "la diferencia no es la negación, sino que lo negativo es por el contrario la diferencia dada vuelta, vista desde el lado pequeño" (1968: 303). En ese mismo sentido la Doctrina de la ciencia no es la identidad engendrada a partir de la oposición, sino que la oposición entre el Yo y el no-Yo es la Doctrina de la ciencia vista del lado pequeño, como hace Hegel. 
Así, el Yo = Yo debe interpretarse desde la perspectiva de la intuición intelectual y no a la inversa, porque de lo contrario se pierde lo que Fichte intenta subrayar cuando, harto de los malentendidos acerca de su sistema, elimina la expresión Yo = Yo de la Nova methodo: que el Yo no se intuye a sí mismo en la actividad de reflexión como forma de lo idéntico. El Yo sólo es puro en tanto autoconciencia inmediata o intuición intelectual; en cuanto la autoconciencia vuelve sobre sí, es decir, en cuanto reflexiona, se escinde (Fichte, 1994a: 34): "si es puesta la conciencia, entonces es puesta la escisión” (Fichte, 1977: 24). En el concepto del Yo se piensan Yo y no-Yo (Fichte, 1994a, 46). Este movimiento apuntala la interpretación de Goddard, quien afirma que "La intuición intelectual no puede dejar de incluir en sí una partición” (1999: 99) y "el yo fichteano es un yo fisurado" (79). El círculo de la reflexión es así un círculo fracasado, inherentemente fracasado. Lo cual nos lleva a pensar que quizás el Yo fichteano, lejos de ser el sujeto, la certeza del cogito, sea la fuerza que deshace el sujeto individual, y hace que el Yo empírico se encuentre de pronto subido a la espalda de un tigre. La Thathandlung no es identidad, sino diferencia.

No se trata por tanto en Fichte de partir de la quieta unidad del Yo consigo mismo (identidad analítica), ni tampoco de establecer como piensa Deleuze la identidad sintética entre el Yo y el no-Yo que aparecen como opuestos, ${ }^{46}$ sino de partir de una actividad ${ }^{47}$ que tiene la forma de círculos que no se cierran sobre sí. Fichte recurre insistentemente (como luego lo hará Hegel) a la figura del círculo para explicar su pensamiento. Pero hay que evitar dos mal-

46 "Los poskantianos acordarán que la identidad analítica es la identidad vacía que sólo les permite pensar la esencia o lo posible. Pero la identidad sintética es la operación por la cual el pensamiento se eleva a la potencia de lo existente y toma posesión de lo existente" (Deleuze, 2011: 588).

47 "Fichte no deja de insistir sobre la importancia de una palabra: sustituir. Se trata de sustituir el hecho de la conciencia por el acto de la conciencia" (Deleuze, 1956). 
entendidos: 1) por "círculo" no debe entenderse el sucederse de figuras a lo largo de la circunferencia, sino la producción del círculo a partir de un centro mediante a una multiplicidad de radios (Fichte, 1994a: 170, 207 y 227); 2) el centro no es ocupado por ninguna síntesis particular, ${ }^{48}$ sino por la intuición intelectual misma. La unificación del Yo no es en realidad más que la proyección de los rayos como figuras de un círculo de la reflexión que nunca termina de cerrar sobre sí mismo. ${ }^{49}$

El centro del círculo no es -no puede ser- figura de una unificación imposible. Y ese es, según Fichte, un rasgo distintivo de la Doctrina de la ciencia respecto al dogmatismo: "[A diferencia del dogmatismo] en la Doctrina de la ciencia el concepto de yo no reside en nosotros, sino que es producido a través de la multiplicidad" (Fichte, 1987: 226; se ha modificado la traducción). El Yo como inmanencia es entonces una multiplicidad (no como "partes", dado que "el Yo no puede ser dado, porque no tiene partes, a las cuales lo dado pudiera estar atado"; Fichte, 1994a: 81), y no una Unidad quieta, idéntica a sí misma, a salvo de toda violencia y segura de su camino recto. En suma: elude todas las trampas de la imagen dogmática del pensamiento. Tampoco "colma lo que separa" en tanto la concordancia entre Yo y Yo nunca es completa, y por lo tanto elude también la acusación de ser "demasiado dogmático" (Cfr. supra, apartado 4). A través de la afirmación de una

${ }^{48}$ Gaudio difiere de esta interpretación, señalando que en la Nova methodo ese lugar lo ocupa la voluntad (2016: 127-128).

${ }^{49}$ Así, a partir de la escisión originaria de la reflexión entre Yo y no-Yo, se multiplican los círculos fracasados: "sujeto y objeto", "Yo absoluto y Yo limitado", actividad real y actividad ideal (Fichte 1994a: 51), conocimiento objetivo y conocimiento de fin (1987: 116), sentimiento y actuar (1994a: 138), efectividad real -concepto de fin- conocimiento (152), ser y pensar (182), cuerpo y conciencia (234), pensar que unifica y pensar que divide (185), mundo y reino de los seres racionales (225), hasta llegar a la quintuplicidad final donde la complejidad del entramado de relaciones recíprocas queda en evidencia. 
"multiplicidad positiva [...] el dogmatismo se queda sin pretextos" (Fichte, 1994a: 68-69, 71), tanto en sentido fichteano como en sentido deleuziano.

\section{Conclusión}

El dogmatismo interpela tanto a Deleuze como a Fichte en los fundamentos de sus respectivas ontologías. En el caso de Deleuze, exige precisar el sentido de la inmanencia cuando su ontología se desdobla en diferentes planos, básicamente, lo actual y lo virtual, y la intensidad y la extensión. En el caso de Fichte, exige comprender que la relación del Yo consigo mismo expresada en la fórmula Yo = Yo no puede ser una identidad, ni remitir a un punto de unificación. De esa manera, se descubre que ninguno de los dos es, en sentido estricto, un dogmático. Sin embargo, en ese vía de interpelación, la filosofía comparada nos ha permitido vivificar la interpretación de las ontologías de ambos autores. El movimiento de producción de las determinaciones de la Doctrina de la ciencia a partir de la Thathandlung es un círculo fracasado, que nunca termina de cerrar sobre sí mismo. Y un círculo fracasado no es otra cosa que un pliegue. A través de esta sucesión de pliegues, la intuición intelectual traza el campo trascendental. Este trazado resulta difícil entender desde la geometría clásica a la que recurre Fichte, pero es ilustrada mejor -aunque siempre de forma insuficiente, representativa- por la topología, a la cual, como vimos, recurre Deleuze. ${ }^{50}$ El pliegue es el movimiento por el cual se constituye la multiplicidad intensiva que es la razón de lo múltiple-empírico. Esa multiplicidad se expresa como el sistema de lo múltiple Yo =

${ }^{50}$ Goddard señala, siguiendo a Merleau-Ponty, que el campo trascendental fichteano debe pensarse desde la geometría moderna: restricción, segregación, modulación. Sin embargo, sigue pensando esa espacialidad como dada, y no construida (Goddard, 1999: 70). 
Yo. El "=" como pliegue se desdobla en un Yo cualitativo, unificado, puro (lo que Fichte llama Yo absoluto) y un Yo cuantitativo, extensivo, como composición de partes (lo que Fichte llama Yo empírico). La clave de la filosofía es ubicarse en ese pliegue, y alcanzar así el punto de vista de la génesis como verdadera condición de la experiencia real.

\section{Bibliografía}

Amrine, Fredrick, 2015, "«The magic formula we all seek»: Spino$\mathrm{za}+$ Fichte $=\mathrm{x}$ ", Craig Lundy y Daniela Voss (eds.), At the Edges of Thought, Deleuze and Post-Kantian Philosophy, Edinburgh University Press, Edinburgh, pp. 168-189.

DeLanda, Manuel, 2010, Deleuze: History and Science, Antropos, Nueva York.

Deleuze, Gilles, 1956, Qu'est-ce que fonder?. Disponible en: http:// www.webdeleuze.com/php/texte.php?cle=218\&groupe $=$ Conf \%E9rences\&langue=1 (Consultado: 5/VII/2016).

, 1963, La philosophie critique de Kant, PUF, París.

, 1968, Différence et répétition, PUF, París.

, 2003, "L'immanence: une vie...", en Deux régimes de fous et autres textes, Minuit, París, pp. 359-363.

,2011, Cine II, Los signos del movimiento y el tiempo, Cactus, Buenos Aires.

,2015a, Lettres et autres textes, Minuit, París.

, 2015b, La subjetivación, curso sobre Foucault, III, Cactus, Buenos Aires.

Deleuze, Gilles y Guattari, Felix, 1980, Mille Plateaux, Minuit, París.

, 1991, ¿Qué es la filosofía?, Minuit, París. 
Descombes, Vincent, 1988, Lo mismo y lo otro, cuarenta y cinco años de filosofía francesa (1933-1978), Elena Benarroch (trad.), Cátedra, Madrid.

Ferrater Mora, José, 1994, Diccionario de la filosofía, t. 1, Ariel, Barcelona.

Ferreyra, Julián, 2016a, "La voluptuosidad de ser otro en el joven Deleuze”, en El banquete de los dioses, vol. 4, núm. 6.

, 2016b, "Deleuze's Bêtise: Dissolution and Genesis in the Properly Human Form of Bestiality", en Comparative and Continental Philosophy, pp. 1-11.

Fichte, Johann, 1998, "Sobre la diferencia entre el espíritu y la letra en filosofía", en Filosofía y estética, Manuel Ramos Valera y Faustino Oncina (trad.), Universidad de Valencia, Valencia, pp. 135-167.

, 1994a, Wissenschaftslehre nova methodo, Manuscrito de Krause, Hamburg, Meiner.

, 1994b, Fundamento del derecho natural según los principios de la Doctrina de la ciencia, José Villacañas Berlanga, Manuel ramos Valera y Faustino Oncina Coves (trads.), Centro de Estudios Constitucionales, Madrid.

, 1987, Doctrina de la Ciencia nova methodo [manuscrito de Halle], José Luis Villacańas y Manuel Ramos (trads.), Natan, Valencia.

, 1977, Das System der Sittenlehre, Frommann, Gesamtausgabe der Bayerischen Akademie, t. 1, núm. 5, Stuttgart.

, 1975, Fundamento de toda la doctrina de la ciencia, en Doctrina de la ciencia, Juan Cruz Cruz (trad.), Aguilar, Buenos Aires.

, 1970a, Erste Einleitung in die Wissenschaftslehre, Frommann, Gesamtausgabe der Bayerischen Akademie, t. I, núm. 4, Stuttgart. 
, 1970b. Zweite Einleitung in die Wissenschaftslehre, Frommann, Gesamtausgabe der Bayerischen Akademie, t. I, núm. 4, Stuttgart.

, 1963, Sobre el concepto de la Doctrina de la ciencia, Bernabé Navarro (trad.), Centro de Estudios Filosóficos, UNAM, México.

, 1845, Die Anweisung zum seligen Leben oder auch die Religionslehre, Veit, Sämmtliche Werke, t. 5, Berlín.

Foucault, Michel, 1966, Les mots et les choses, Gallimard, París.

Fuchs, Erich, 1994, "Einleitung”, en Johann Fichte, Wissenschaftslehre nova methodo, Meiner, Hamburg.

Hegel, Georg, 2010, Diferencia entre los sistemas de filosofía de Fichte y Schelling, María del Carmen Paredes Martín (trad.), Gredos, Madrid.

, 1970, Enzyklopädie der philosophischen Wissenschaften, Werke, t. 8, Suhrkamp, Frankfurt.

Gaudio, Mariano, 2016, La concepción del Estado en el Fundamento del derecho natural de Fichte, tesis doctoral defendida en marzo de 2016, FFyL, UBA, Buenos Aires.

Goddard, Jean-Christophe, 1999, La philosophie fichtéenne de la vie, Vrin, París.

Hammacher, Klaus, 1992, "Fichte und Spinoza”, M. Walther, Königshausen \& Neumann, Würzburg, pp. 81-107.

Ivaldo, Marco, 1992, "Transzendentalphilosophie und "realistische» metaphysik, Das Fichtesche Spinoza-Veständnis”, M. Walther, Königshausen \& Neumann, Würzburg, pp. 59-79.

Kant, Immanuel, 2009, Crítica de la razón pura, Mario Caimi (trad.), Colihue, Buenos Aires. 
1999, Prolegómenos a toda metafísica futura que haya de poder presentarse como ciencia, Mario Caimi (trad.), Itsmo, Madrid.

Krtolica, Igor, 2015, Gilles Deleuze, PUF, París.

Lapoujade, David, 2015, Deleuze, Les mouvements aberrants, Minuit, París.

Solé, María Jimena, 2013, "Spinoza en el origen del Idealismo alemán: Repercusiones de la "Polémica del spinozismo", en Fichte y Schelling", El arco y la lira, núm. 1, pp. 39-54.

Recibido: 23 de marzo de 2017 Aceptado: 20 de mayo de 2017 